\title{
High-Flow Nasal Cannula Compared With Conventional Oxygen Therapy or Noninvasive Ventilation Immediately Postextubation: A Systematic Review and Meta-Analysis
}

\author{
David Granton, $\mathrm{MD}^{1}$; Dipayan Chaudhuri, $\mathrm{MD}^{1}$; Dominic Wang, $\mathrm{HBSc}^{2}$; Sharon Einav, $\mathrm{MD}^{3,4}$; \\ Yigal Helviz, $\mathrm{MD}^{3}$; Tommaso Mauri, $\mathrm{MD}^{5}$; Jordi Mancebo, $\mathrm{MD}^{6}$; Jean-Pierre Frat, $\mathrm{MD}^{7-9}$; \\ Sameer Jog, $\mathrm{MD}^{10}$; Gonzalo Hernandez, MD ${ }^{11}$; Salvatore M. Maggiore, MD ${ }^{12,13}$; Carol L. Hodgson, PhD ${ }^{14}$; \\ Samir Jaber, $\mathrm{MD}^{15}$; Laurent Brochard, $\mathrm{MD}^{16}$; Vatsal Trivedi, MD ${ }^{16}$; Jean-Damien Ricard, $\mathrm{MD}^{17,18}$; \\ Ewan C. Goligher, MD ${ }^{16,19}$; Karen E. A. Burns, MD ${ }^{16,20}$; Bram Rochwerg, MD $^{1,20}$
}

'Department of Medicine, McMaster University, Hamilton, ON, Canada. ${ }^{2}$ Schulich School of Medicine, Western University, London, ON, Canada. ${ }^{3}$ General Intensive Care Unit, Shaare Zedek Medical Center, Jerusalem, Israel. ${ }^{4}$ Faculty of Medicine, Hebrew University, Jerusalem, Israel.

${ }^{5}$ Department of Anesthesia, Critical Care and Emergency, Fondazione IRCCS Ca' Granda Ospedale Maggiore Policlinico, University of Milan, Milan, Italy.

${ }^{6}$ Servei de Medicina Intensiva, Hospital Universitari Sant Pau, Barcelona, Spain.

${ }^{7} \mathrm{CHU}$ de Poitiers, Médecine Intensive Réanimation, Poitiers, France.

IINSERM, CIC-1402, équipe ALIVE, Poitiers, France.

'Université de Poitiers, Faculté de Médecine et de Pharmacie de Poitiers, Poitiers, France.

${ }^{10}$ Department of Intensive Care Medicine, Deenanath Mangeshkar Hospital and Research Centre, Pune, India.

${ }^{11}$ Intensive Care Unit, Hospital Infanta Sofía, Madrid, Spain.

${ }^{12}$ Department of Medical, Oral and Biotechnological Sciences, Gabriele d'Annunzio University of Chieti-Pescara, Chieti, Italy.

${ }^{13}$ Department of Anesthesiology and Critical Care, SS. Annunziata Hospital, Chieti, Italy.

${ }^{14}$ Australian and New Zealand Intensive Care Research Centre, Department of Epidemiology and Preventive Medicine, Monash University, Melbourne, VIC, Australia.

${ }^{15}$ Department of Anaesthesiology and Critical Care Medicine B (DAR B), Saint-Eloi Teaching Hospital, PhyMed Exp, INSERM U1046, University of Montpellier, Montpellier, France.

${ }^{16}$ Interdepartmental Division of Critical Care Medicine, University of Toronto, Toronto, ON, Canada.

${ }^{17}$ Assistance Publique-Hôpital de Paris, Service de Réanimation Médicochirurgicale, Hôpital Louis Mourier, Colombes, France.

${ }^{18}$ Université de Paris, INSERM, IAME, UMR 1137, Paris, France.

${ }^{19}$ Department of Medicine, Division of Respirology, University Health Network, Toronto, ON, Canada.

${ }^{20}$ Department of Health Research Methods, Evidence and Impact, McMaster University, Hamilton, ON, Canada.
Objectives: Reintubation after failed extubation is associated with increased mortality and longer hospital length of stay. Noninvasive oxygenation modalities may prevent reintubation. We conducted a systematic review and meta-analysis to determine the safety and efficacy of high-flow nasal cannula after extubation in critically ill adults.

Data Sources: We searched MEDLINE, EMBASE, and Web of Science.

Study Selection: We included randomized controlled trials comparing high-flow nasal cannula to other noninvasive methods of oxygen delivery after extubation in critically ill adults.

Data Extraction: We included the following outcomes: reintubation, postextubation respiratory failure, mortality, use of noninvasive ventilation, ICU and hospital length of stay, complications, and comfort.

Data Synthesis: We included eight randomized controlled trials ( $n=1,594$ patients). Compared with conventional oxygen therapy, high-flow nasal cannula decreased reintubation (relative risk, 0.46; 95\% Cl, 0.30-0.70; moderate certainty) and postextubation respiratory failure (relative risk, $0.52 ; 95 \% \mathrm{Cl}, 0.30-0.91$; very low certainty), but had no effect on mortality (relative risk, $0.93 ; 95 \% \mathrm{Cl}, 0.57-1.52$; moderate certainty), or ICU length of stay (mean difference, $0.05 \mathrm{~d}$ fewer; $95 \% \mathrm{Cl}, 0.83 \mathrm{~d}$ fewer to $0.73 \mathrm{~d}$ more; high certainty). High-flow nasal cannula may decrease use of noninvasive ventilation (relative risk, 0.64; 95\% $\mathrm{Cl}, 0.34-1.22$; moderate certainty) and hospital length of stay (mean difference, $0.98 \mathrm{~d}$ fewer; $95 \% \mathrm{Cl}, 2.16 \mathrm{~d}$ fewer to 0.21 $\mathrm{d}$ more; moderate certainty) compared with conventional oxygen therapy, however, certainty was limited by imprecision. Compared with noninvasive ventilation, high-flow nasal cannula had no effect on reintubation (relative risk, $1.16 ; 95 \% \mathrm{Cl}, 0.86-1.57$; low certainty), mortality (relative risk, 1.12; 95\% Cl, 0.82-1.53; moderate certainty), or postextubation respiratory failure (relative risk, 0.82 ; 
95\% Cl, 0.48-1.41; very low certainty). High-flow nasal cannula may reduce ICU length of stay (moderate certainty) and hospital length of stay (moderate certainty) compared with noninvasive ventilation.

Conclusions: High-flow nasal cannula reduces reintubation compared with conventional oxygen therapy, but not compared with noninvasive ventilation after extubation. (Crit Care Med 2020; 48:e1129-e1136)

Key Words: high-flow nasal cannula; meta-analysis; noninvasive ventilation; oxygen inhalational therapy; postextubation; reintubation

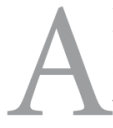

lthough invasive mechanical ventilation can be lifesaving, weaning and ensuring sustained separation from ventilatory support can be challenging. Complications are common in the postextubation period, especially reintubation, which has been associated with increased mortality, longer hospital length of stay (LOS), and increased costs (1). A large prospective cohort study determined the rate of reintubation to be approximately $10 \%$ across diverse ICUs (1); however, other studies have reported reintubation rates as high as $20 \%(2)$.

Once postextubation respiratory failure develops, treatment with noninvasive ventilation (NIV) has been associated with worse outcomes compared with reintubation (3). Multiple strategies to prevent development of respiratory failure in the postextubation setting have been attempted. Preventative strategies, applied immediately after extubation, include conventional oxygen therapy (COT) (nasal prongs or Venturi mask), NIV (continuous positive airway pressure or bilevel positive airway pressure), or high-flow nasal cannula (HFNC). COT is typically limited to oxygen flow less than or equal to $15 \mathrm{~L} / \mathrm{min}$, while HFNC can deliver flow up to $50-60 \mathrm{~L} / \mathrm{min}$ of heated and humidified oxygen $(4,5)$. HFNC can washout pharyngeal dead space, reduce respiratory resistance, provide some positive end-expiratory pressure $\left(2-8 \mathrm{~cm} \mathrm{H}_{2} \mathrm{O}\right)(6)$, and may facilitate mucus clearance. Compared with COT, HFNC may be noninferior in regards to dyspnea and patient comfort (7). Compared with NIV, HFNC may be more comfortable and better tolerated by patients (8).

Previous systematic reviews have combined trials evaluating HFNC in critically ill patients in the ICU and postoperative patients (9-12). This approach is problematic as surgical patients are typically not hypoxemic, are not suffering from acute respiratory illnesses, and have different clinical trajectories as compared with patients who are critically ill. Surgical patients are also typically intubated for a shorter period of time (12). These factors, individually and collectively, limit the generalizability of findings from prior systematic reviews to critically ill patients that have not recently undergone surgery. Previous systematic reviews examining HFNC use in the postextubation setting have demonstrated inconsistent results. Although some systematic reviews have shown a reduction in reintubation rate with HFNC (10-12) when compared with
COT, others have not (9). These disparate findings may reflect the clinical heterogeneity of the included trials. We sought to perform a systematic review and meta-analysis examining the role of HFNC compared with COT or NIV after extubation in critically ill patients only.

\section{MATERIALS AND METHODS}

We registered this systematic review and meta-analysis on PROSPERO (Number CRD42019136636) and used the Preferred Reporting Items for Systematic Reviews and Meta-Analyses checklist to report findings (Supplementary Table 1, Supplemental Digital Content 1, http://links.lww.com/CCM/F768).

\section{Data Sources and Searches}

We performed a search using MEDLINE, EMBASE, and Web of Science from January 2007 to October 8, 2019. We did not search prior to 2007 as HFNC use was infrequent before this time. We restricted our search to articles published in English or Spanish. We searched studies of adults treated with HFNC using keywords "humans" together with "adult," "mature," or "grown" and "high flow nasal cannula," "high flow nasal therapy," "high flow nasal oxygen," "high flow oxygen therapy," "high flow therapy," "optiflow (respiration)," and "nasal highflow." We did not exclude trials published in abstract form only or based on trial quality.

\section{Trial Selection}

Three reviewers (D.G., D.C., D.W.), working in pairs, screened trials independently and in duplicate. Disagreements were resolved by discussion and third party adjudication (B.R.). We screened citations in two steps, initially based on title and abstract, and then by full text. We captured reasons for trial exclusion at the full manuscript review stage.

We included randomized controlled trials (RCTs) that compared HFNC to either COT or NIV during the immediate postextubation period in critically ill patients admitted to ICU. We excluded case reports, case series, observational studies, and studies exclusively involving patients who were extubated after general anesthesia for elective surgery, including patients admitted to the ICU for a short time period $(<24 \mathrm{hr})$ following cardiothoracic surgery. We examined the following outcomes: reintubation, mortality, use of NIV (only applicable in COT and HFNC groups), ICU LOS, hospital LOS, patient comfort (as defined by trial authors), postextubation respiratory failure (as defined by trial authors), and complications of therapy. If multiple time points were presented for mortality or reintubation, we used the measurement at the time furthest from initial extubation.

\section{Data Extraction and Quality Assessment}

Three reviewers (D.G., D.C., D.W.), working in pairs, performed data abstraction independently and in duplicate using a standardized data abstraction form. A fourth author (B.R.) resolved disagreements, where needed. We collected data including trial characteristics, patient demographics, HFNC and comparator parameters, outcome data, as well as individual trial risk 
of bias (ROB). If only median and interquartile range were reported for a given continuous outcome, we assumed a normal distribution (i.e., median $=$ mean) and interquartile range was converted to SD using methods described by the Cochrane Collaboration (13). We assessed ROB in duplicate using the modified Cochrane ROB tool (14). This tool assesses sequence generation, allocation concealment, blinding, incomplete data, selective reporting, and other bias. ROB was labeled as "low," "probably low," "probably high," or "high" per domain. Overall trial ROB was determined based by the highest risk in any given ROB domain, excluding ROB arising from lack of blinding. Given the nature of the intervention and comparator, it was presumed that ROB related to blinding would be high across all included trials. ROB related to outcome assessment for the outcome of reintubation was also presumed to be high across trials given subjectivity in decision-making regarding reintubation (15). We assessed overall certainty of evidence for each outcome using the Grading of Recommendations, Assessment, Development, and Evaluation (GRADE) framework (16).

\section{Data Analysis}

We performed all statistical analyses in RevMan 5.3 (The Nordic Cochrane Centre, The Cochrane Collaboration, Copenhagen, 2014). We used the DerSimonian-Laird random-effects

Search "high flow nasal cannul*" etc' as in text AND (adult OR mature OR Grown) Filters: Publication date from 2007/01/01 to 2019/10/09; Humans; English; Spanish

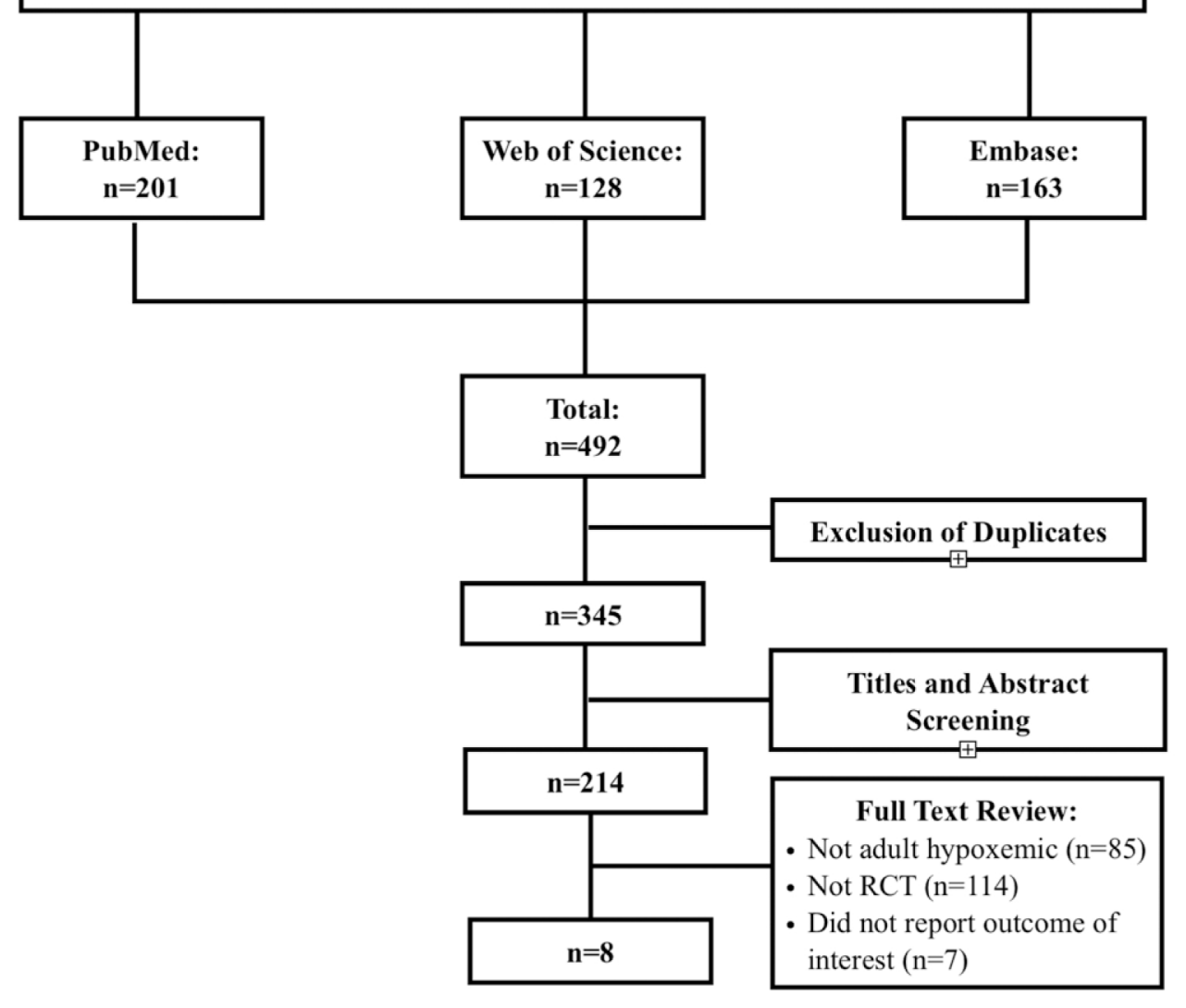

Figure 1. Preferred Reporting Items for Systematic Reviews and Meta-Analyses flow-study inclusion. RCT = randomized controlled trial. model to pool trial data, with trial weighting determined by the inverse-variance method. We present summary estimates of effect using mean difference (MD) for continuous outcomes and risk ratio for dichotomous outcomes, both with 95\% CIs. We assessed for statistical heterogeneity using the chi-square test for homogeneity, the $I^{2}$ statistic (17), and visual inspection of the forest plots. We assessed heterogeneity in RCT methodology by comparing patient populations, intervention and control parameters, and variability of outcome definitions, measurement, and reporting through GRADE assessment.

We identified four a priori subgroups of interest: high versus low risk of extubation failure (as defined by trial authors), prophylaxis versus treatment of postextubation respiratory failure, patients with versus without a history of chronic obstructive pulmonary disease (COPD), and high ROB versus low ROB trials. We hypothesized that HFNC would be more effective when used prophylactically to prevent postextubation respiratory failure, and in trials at high (vs low) ROB, when compared with either COT or NIV. We hypothesized HFNC to be more effective in patients at high risk of extubation failure and in those with a history of COPD when compared with COT and less effective when compared with NIV. A post hoc sensitivity analysis was performed excluding trials that used continuous positive airway pressure as a comparator. At request of the peer reviewers, we performed an additional post hoc sensitivity analysis excluding three trials; one which was only available in abstract form (18), one randomized crossover design (19), and one including exclusively COPD patients (20).

\section{RESULTS}

\section{Search Strategy and Trial Characteristics}

We reviewed 492 citations, with eight RCTs meeting eligibility criteria $(n=1,594$ patients total) (18-25). The eight RCTs included seven full manuscript publications (1925) and one abstract (18) (Fig. 1). We present individual trial characteristics in (Supplementary Table 2, Supplemental Digital Content 1, http://links. lww.com/CCM/F768). Trials included 17 to 600 randomized patients. All trials used noninvasive oxygenation strategies as prophylactic and not as treatment of postextubation respiratory failure. One trial used a randomized, crossover 
design (19) for which we were only able to include shortterm outcome data related to comfort. Three trials examined patients who were deemed to be at high risk for extubation failure $(18,24,25)$, whereas the remaining five trials were either low or unspecified risk for extubation failure (19-23). Five trials compared HFNC to COT (i.e., nasal cannula, Venturi mask, or nonrebreather mask) $(19,21-23,25)$, whereas the remaining three compared $\operatorname{HFNC}$ to $\operatorname{NIV}(18,20,24)$ applied immediately following extubation. Only one trial included exclusively COPD patients, in which COPD exacerbation was the initial reason for intubation (20).

Reintubation rates were reported at 24 hours following extubation in one trial (21) and at 72 hours following extubation in three trials (23-25). In one trial, reintubation was recorded throughout the remainder of the ICU admission (22). Timing of reintubation was not reported in two trials $(18,20)$. Many of the trials reported criteria for reintubation which were fairly consistent including clinical features such as cardiac or respiratory arrest, changes in mental status, respiratory failure, copious secretions, massive aspiration, need for emergency surgery, and hemodynamic collapse. The minimum duration of invasive mechanical ventilation prior to randomization in the included trials was variable: with three trials using at least 12 hours (23-25), one trial using a 24-hour threshold (22), and another trial using a minimum of 48 hours (21). Two trials reported that the mean duration of mechanical ventilation (19, 20) prior to randomization was at least 48 hours.

The HFNC devices used included Optiflow (Fisher \& Paykel Healthcare, Auckland, New Zealand) (19, 20, 22-25), AIRVO 2 (Fisher \& Paykel Healthcare) (20), or PT101AZ (Fisher \& Paykel Healthcare) (21). One trial (18) did not specify the HFNC device used. All trials featured either a fixed flow rate (22) or mean flow rate $(19-21,23,24)$ ranging between 30 and $55 \mathrm{~L} / \mathrm{min}$. Two trials did not report the specific flows used (18, 25). The $\mathrm{FIO}_{2}$ used was variable across trials, being set to 0.4 in one trial (21), featuring a mean value of $0.3-0.4$ in the others $(20,22-24)$ and not described in three trials $(18,19,25)$.

We summarize assessments of individual trial ROB in Supplementary Table 3 (Supplemental Digital Content 1, http://links.lww.com/CCM/F768). No trial blinded patients or clinicians to the devices used. Other than the blinding domain, only one trial was assessed to be at high ROB (18). The remaining trials were judged to be low or probably low ROB.

\section{Outcomes}

We present summary estimates of effect of HFNC versus COT and NIV on outcomes in Supplementary Table 4 (Supplemental Digital Content 1, http://links.lww.com/CCM/F768) with GRADE ratings. We provide forest plots comparing HFNC versus COT or NIV on postextubation respiratory failure, NIV use, ICU and hospital LOS, and patient comfort outcomes in Supplementary Figure 1 (Supplemental Digital Content 2, http://links.lww.com/CCM/F769 [legend, Supplemental Digital Content 1, http://links.lww.com/CCM/F768]), Supplementary Figure 2 (Supplemental Digital Content 3, http://links. lww.com/CCM/F770 [legend, Supplemental Digital Content 1,
http://links.lww.com/CCM/F768]), Supplementary Figure 3 (Supplemental Digital Content 4, http://links.lww.com/CCM/ F771 [legend, Supplemental Digital Content 1, http://links.lww. com/CCM/F768]), Supplementary Figure 4 (Supplemental Digital Content 5, http://links.lww.com/CCM/F772 [legend, Supplemental Digital Content 1, http://links.lww.com/CCM/ F768]), Supplementary Figure 5 (Supplemental Digital Content 6, http://links.lww.com/CCM/F772 [legend, Supplemental Digital Content 1, http://links.lww.com/CCM/F768]), and Supplementary Figure 6 (Supplemental Digital Content 7, http://links. lww.com/CCM/F773 [legend, Supplemental Digital Content 1, http://links.lww.com/CCM/F768]).

\section{HFNC Versus COT}

Compared with COT, HFNC decreased the rate of reintubation (relative risk [RR], 0.46; 95\% CI, 0.30-0.70; 8.1\% absolute risk reduction $[\mathrm{ARR}]$; $95 \% \mathrm{CI}, 10.5-4.5 \%$ reduction; moderate certainty) (Fig. 2), and incidence of post extubation respiratory failure (RR, 0.52; 95\% CI, 0.30-0.91; 9.4\% ARR; 95\% CI, 13.7$1.8 \%$ reduction; very low certainty) but had no effect on mortality (at the longest follow-up point reported) ( $\mathrm{RR}, 0.93 ; 95 \% \mathrm{CI}$, $0.57-1.52 ; 0.5 \%$ ARR; $95 \%$ CI, 3.3\% reduction to $4.0 \%$ increase; moderate certainty) (Fig. 3) or ICU LOS (MD, 0.05 d fewer; 95\% CI, $0.83 \mathrm{~d}$ fewer to $0.73 \mathrm{~d}$ more; high certainty). HFNC may decrease the use of NIV (RR, 0.64; 95\% CI, 0.34-1.22; 5.2\% ARR; 95\% CI, 9.5\% reduction to $3.2 \%$ increase; moderate certainty) and shorten hospital LOS (MD, $0.98 \mathrm{~d}$ fewer; 95\% CI, $2.16 \mathrm{~d}$ fewer to $0.21 \mathrm{~d}$ more; moderate certainty), however, certainty in both of these outcomes was limited by imprecision. HFNC use may improve patient comfort as measured by a Visual Analog Scale (MD, -2.25 ; 95\% CI, -5.68 to 1.18 ; low certainty).

\section{HFNC Versus NIV}

Compared with NIV, HFNC had no effect on the rate of reintubation (RR, 1.16; 95\% CI, 0.86-1.57; 2.8\% absolute risk increase; $95 \% \mathrm{CI}, 2.5 \%$ reduction to $10.0 \%$ increase; low certainty) (Fig. 2), mortality (at the longest follow-up point reported) (RR, 1.12; 95\% CI, 0.82-1.53; 2.2\% absolute risk increase; $95 \% \mathrm{CI}, 3.3 \%$ reduction to $9.7 \%$ increase; moderate certainty) (Fig. 3), or postextubation respiratory failure (RR, 0.82; 95\% CI, $0.48-1.41 ; 6.3 \%$ ARR; 95\% CI, $18.1 \%$ reduction to $14.2 \%$ increase; very low certainty). However, HFNC reduced ICU LOS (MD, 0.99 d fewer; 95\% CI, 1.68-0.30 d fewer; moderate certainty) and improved patient comfort (MD, -1.60; $95 \% \mathrm{CI},-2.88$ to -0.32 ; moderate certainty). HFNC may decrease hospital LOS (MD, 3.0 d fewer; 95\% CI, $6.24 \mathrm{~d}$ fewer to $0.24 \mathrm{~d}$ more; moderate certainty).

We did not conduct planned subgroup analyses related to a history of COPD or indication for treatment (treatment vs prophylaxis) due to lack of data reporting these subgroups. ROB subgroup analysis was only performed for the reintubation and postextubation respiratory failure outcomes in the NIV comparator due to lack of available data for other outcomes. We did not identify credible subgroup effects based on ROB (Supplementary Fig. 7, Supplemental Digital Content 8, http://links.lww.com/CCM/F774 [legend, Supplemental 


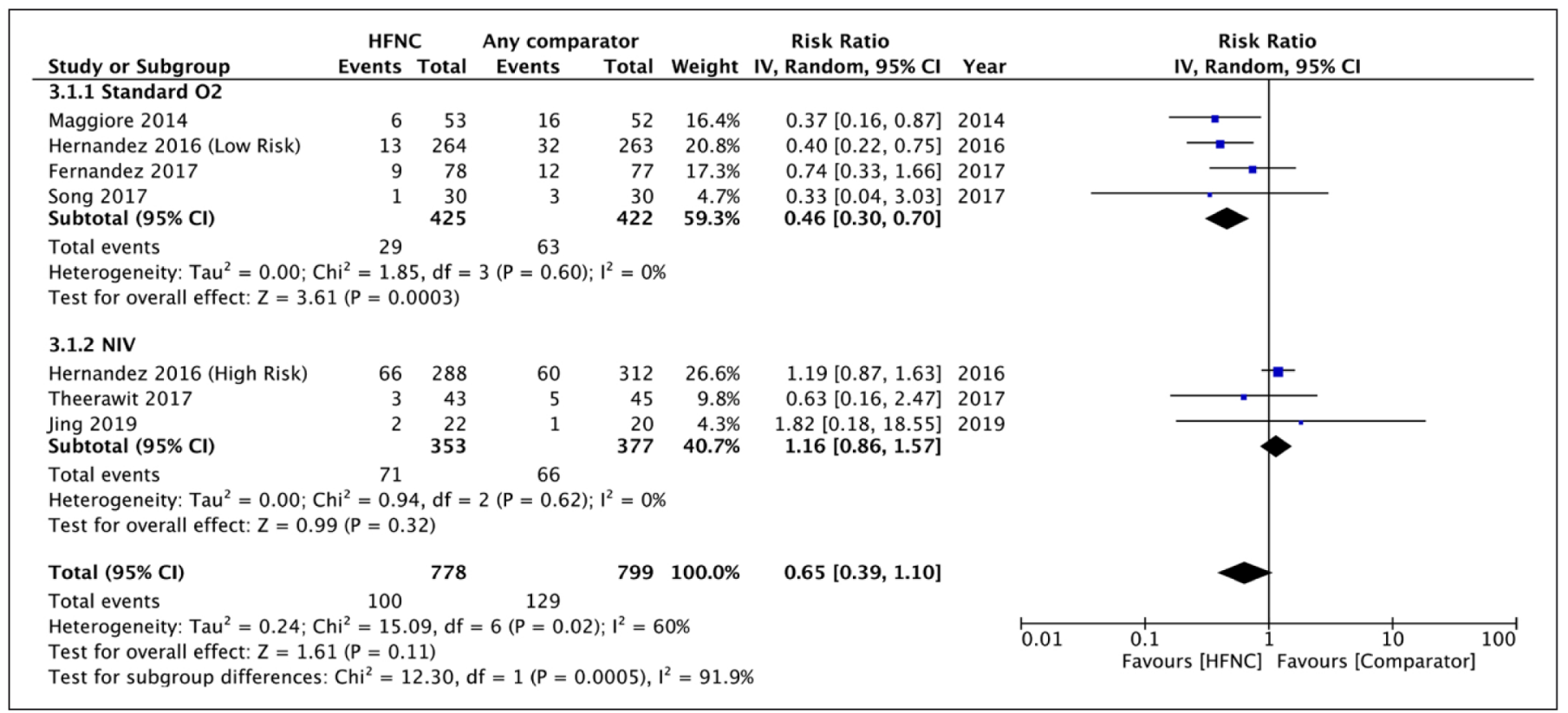

Figure 2. Reintubation forest plot. $d f=$ degrees of freedom, HFNC = high-flow nasal cannula, NIV = noninvasive ventilation.

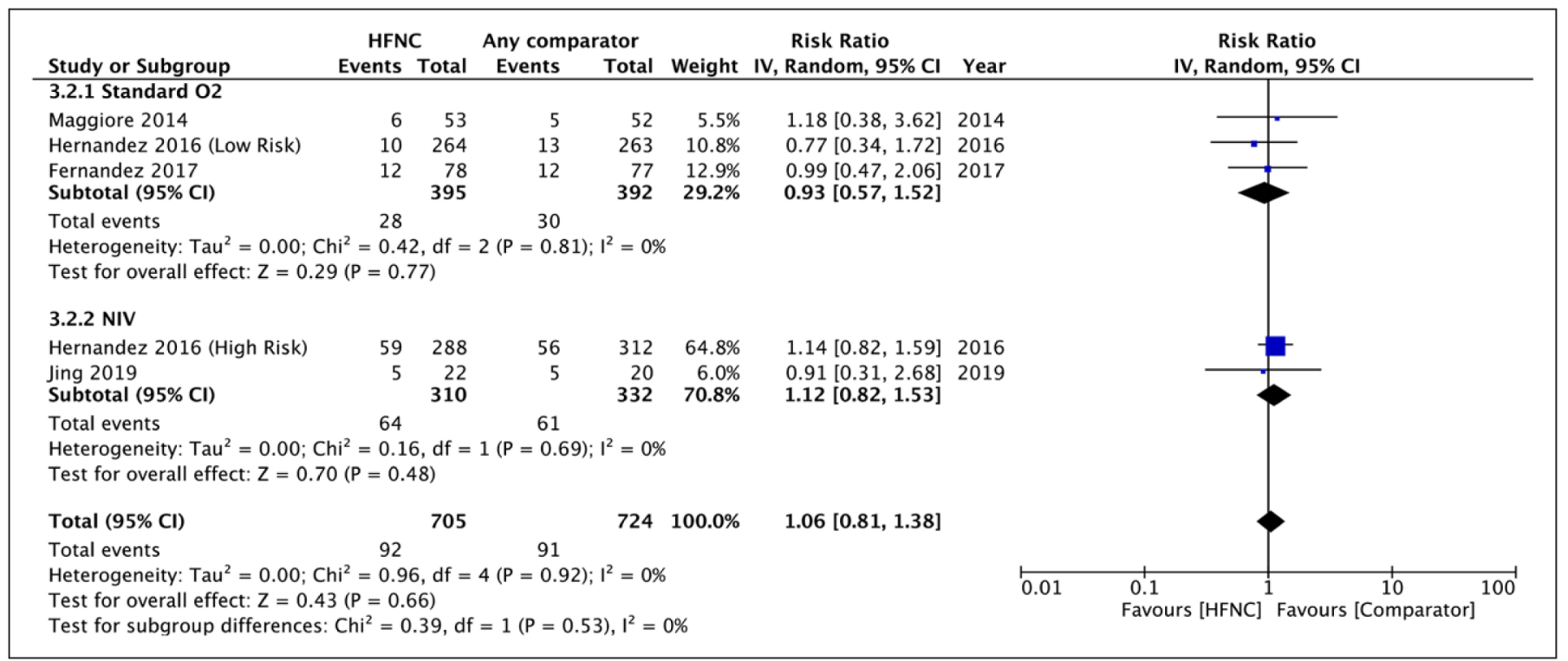

Figure 3. Mortality forest plot. $d f=$ degrees of freedom, HFNC = high-flow nasal cannula, NIV = noninvasive ventilation.

Digital Content 1, http://links.lww.com/CCM/F768]) and Supplementary Fig. 8, Supplemental Digital Content 9, http:// links.lww.com/CCM/F775 [legend, Supplemental Digital Content 1, http://links.lww.com/CCM/F768]) or risk of extubation failure (Supplementary Fig. 8, Supplemental Digital Content 9, http://links.lww.com/CCM/F775; Supplementary Fig. 9, Supplemental Digital Content 10, http://links.lww.com/CCM/ F776; Supplementary Fig. 10, Supplemental Digital Content 11, http://links.lww.com/CCM/F777; Supplementary Fig. 11, Supplemental Digital Content 12, http://links.lww.com/CCM/ F778; Supplementary Fig. 12, Supplemental Digital Content 13, http://links.lww.com/CCM/F779; Supplementary Fig. 13, Supplemental Digital Content 14, http://links.lww.com/CCM/ F780; Supplementary Fig. 14, Supplemental Digital Content 15,
http://links.lww.com/CCM/F781; Supplementary Fig. 15, Supple mental Digital Content 16, http://links.lww.com/CCM/F782; Supplementary Fig. 16, Supplemental Digital Content 17, http:// links.lww.com/CCM/F783; Supplementary Fig. 17, Supplemental Digital Content 18, http://links.lww.com/CCM/F784; Supplementary Fig. 18, Supplemental Digital Content 19, http:// links.lww.com/CCM/F785 [legend, Supplemental Digital Content 1, http://links.lww.com/CCM/F768]) for any of the outcomes of interest for either comparison. Sensitivity analysis excluding studies which used continuous positive airway pressure as a comparator (19) had no credible effect on outcomes of interest (Supplementary Fig. 19, Supplemental Digital Content 20, http://inks.lww.com/CCM/ F786; Supplementary Fig. 20, Supplemental Digital Content 21, http://links.lww.com/CCM/F787 [legend, Supplemental Digital 
Content 1, http://links.lww.com/CCM/F768]). Sensitivity analysis excluding one abstract, one crossover RCT, and one trial of exclusively COPD patients (18-20) showed no credible effect for any outcomes of interest (Supplementary Fig. 21, Supplemental Digital Content 22, http://links.lww.com/CCM/ F788; Supplementary Fig. 22, Supplemental Digital Content 23, http://links.lww.com/CCM/F789; Supplementary Fig. 23, Supplemental Digital Content 24, http://links.lww.com/CCM/ F790; Supplementary Fig. 24, Supplemental Digital Content 25, http://links.lww.com/CCM/F791; Supplementary Fig. 25, Supplemental Digital Content 26, http://links.lww.com/CCM/ F792; Supplementary Fig. 26, Supplemental Digital Content 27, http://links.lww.com/CCM/F793 [legend, Supplemental Digital Content 1, http://links.lww.com/CCM/F768]). Complications were variably reported in the included trials and precluded pooling. Due to this heterogeneity, we have instead summarized the complications reported in the included trials in Supplementary Table 5 (Supplemental Digital Content 1, http://links.lww.com/CCM/F768).

\section{DISCUSSION}

Our systematic review and meta-analysis suggests that HFNC use is associated with reduced rates of reintubation and postextubation respiratory failure in critically ill ICU patients compared with COT, but not NIV. HFNC also may reduce ICU LOS when compared with NIV, but not COT. Subgroup analyses examining patients with a high versus low risk of extubation failure or high versus low ROB did not reveal credible subgroup effects. Further preplanned subgroup analyses were not possible due to lack of granular data. Sensitivity analysis excluding studies using continuous positive airway pressure devices as opposed to NIV did not alter conclusions. Sensitivity analysis excluding one abstract, one crossover RCT, and one trial of exclusively COPD patients (18-20) did not change conclusions for any outcomes of interest (Supplementary Fig. 21, Supplemental Digital Content 22, http://links.lww.com/CCM/F788; Supplementary Fig. 22, Supplemental Digital Content 23, http://links.lww.com/CCM/ F789; Supplementary Fig. 23, Supplemental Digital Content 24, http://links.lww.com/CCM/F790; Supplementary Fig. 24, Supplemental Digital Content 25, http://links.lww.com/CCM/F791; Supplementary Fig. 25, Supplemental Digital Content 26, http:// links.lww.com/CCM/F792; Supplementary Fig. 26, Supplemental Digital Content 27, http://links.lww.com/CCM/F793 [legend, Supplemental Digital Content 1, http://links.lww.com/ CCM/F768]).

Previous systematic reviews on this topic have shown inconsistent results. Three previous systematic reviews (10-12) reported that HFNC reduced rates of reintubation when compared with COT, but not when compared with NIV. Only one of these three systematic reviews performed a subgroup analysis based on postoperative status; however, no subgroup effect was identified (12). In contrast to our findings, a recent systematic review suggested that when compared with COT, HFNC did not reduce reintubation rate (9). However, subgroup analysis in this review was limited to patients after cardiac surgery, as opposed to a broader "postoperative" patient cohort (9). Differences in the populations examined appear to be the most likely explanation for the discordant findings between systematic reviews.

The finding that HFNC may reduce reintubation when compared with COT is consistent with our previous work, which showed that HFNC may reduce the need for intubation in patients with hypoxemic respiratory failure (5). We postulate a number of potential mechanisms for this effect including: the ability of HFNC to provide a small amount of positive end-expiratory pressure which may facilitate alveolar recruitment and improved ventilation-perfusion matching, delivery of higher $\mathrm{FiO}_{2}$, provision of higher gas flows more adequately matching patient's inspiratory demands, and decreased work of breathing with HFNC which may prevent diaphragmatic fatigue or injury $(4,26)$.

The observed shorter ICU LOS with the use of HFNC compared with NIV is important as this may facilitate transfer of patients from the ICU to less acute care settings. This has favorable implications for both ICU capacity and cost-effectiveness. Although no specific cost analysis studies have been conducted in this patient population, there is evidence in pediatric populations that HFNC may be less costly than NIV (27). The average daily cost of an ICU stay across all Canadian adult ICUs has been estimated to be $\$ 3,592$ per day (28). Therefore, decreasing ICU LOS may provide clinical and economic benefits.

Assessment of patient comfort was limited due to variable or incomplete outcome reporting among the included trials, as well as, imprecision in pooled estimates of effect. Although only reported in one trial (20), the improved comfort noted with HFNC when compared with NIV is consistent with previous trials evaluating patients with hypoxemic respiratory failure (8). Potential explanations for these findings may include better humidification of air (4) and a preserved ability to speak and tolerate oral intake with HFNC compared with NIV.

This systematic review and meta-analysis have a number of strengths. We limited clinical heterogeneity among included studies by focusing on patients extubated following an episode of acute illness and excluding trials that examined HFNC in the immediate postoperative setting. We used a comprehensive search strategy, including recently published trials, undertook dual and independent screening and data abstraction, adhered to preregistered methodology, and assessed certainty of outcomes using the GRADE approach. Related to these decisions, and in contrast to a recently reported meta-analysis examining a similar topic (9), we demonstrate a reduction in the incidence of reintubation with HFNC as compared with COT. Our review also has limitations. First, despite attempts to limit clinical heterogeneity, the included trials evaluated heterogeneous patient populations and used variable definitions of outcomes (e.g., postextubation respiratory failure) and variable measuring and reporting of outcomes (e.g., time at which reintubation was assessed). Of interest, three trials in our review specifically included some postoperative patients who met trial inclusion criteria (22-24). Heterogeneity among trials also exists based on differences in trial design and lack of detail pertaining to protocol implementation, allowing for variability to arise in clinician decision-making. This heterogeneity is compounded by 
absent or incomplete reporting of clinician adherence to protocol. Second, by necessity, treatments were not blinded in any of the included trials. Third, of the eight RCTs identified, one was a randomized crossover design, one included exclusively patients with COPD, and another was only available in abstract form; limiting the data that could be analyzed from these three trials. Fourth, outcomes were variably or infrequently (e.g., complication rates) reported. Fifth, different criteria were used to classify individuals at high versus low risk for reintubation between trials, none of which have been validated. Sixth, we restricted our search to trials published in English or Spanish. Finally, the included trials used variable criteria for reintubation.

\section{CONCLUSIONS}

The use of HFNC in the postextubation setting reduces the rates of reintubation when compared with COT, but not when compared with NIV.

\section{ACKNOWLEDGMENTS}

This article was written as an initiative of the Pleural Pressure Working Group (https://www.plugwgroup.org), a working group of the Acute Respiratory Failure section of the European Society of Intensive Care Medicine.

\begin{abstract}
Dr. Mauri received funding from Fisher \& Paykel, Drager, and Mindray. Dr. Mancebo received funding from Faron, Medtronic, Janssen; he received support from Covidien (Medtronic) and Canadian Institutes of Health Research (sponsoring a trial); he received support from Fisher \& Paykel and A-Lung provided medical equipment for multicenter trials (high-flow nasal oxygen therapy and extracorporeal $\mathrm{Co}_{2}$ removal respectively); and he received support from IMT Medical provided travel and hotel expenses to attend a meeting. Dr. Frat received personal fees for lectures and reimbursement of travel and accommodations for medical meetings from Fisher \& Paykel Healthcare; and he received personal fees from SOS oxygene. Dr. Maggiore is the principal investigator of the RINO trial (ClinicalTrials.gov, NCT02107183), which was supported by Fisher \& Paykel Healthcare, and received lecture fees from Draeger Medical and General Electric Healthcare. Dr. Hodgson is supported by the Australian Heart Foundation Fellowship and a National Health and Medical Research Council Investigator Grant. Dr. Jaber received funding from Fisher \& Paykel, Drager, Medtronic, Baxter, and Fresenius-Xenios. Dr. Brochard's institution received funding from Medtronic/Covidien, Fisher \& Paykel, and General Electric. Dr. Trivedi received funding from Fisher \& Paykel. Dr. Ricard received travel expenses coverage from Fisher \& Paykel Healthcare to attend scientific meetings; he received support from Fisher \& Paykel Healthcare provided support for the ongoing High-Flow Acute-on-Chronic Respiratory Failure trial (NCT03406572). Dr. Goligher received funding from Getinge. Dr. Rochwerg is supported by a Hamilton Health Sciences Early Career Research Award. The remaining authors have disclosed that they do not have any potential conflicts of interest.
\end{abstract}

\section{REFERENCES}

1. Miltiades AN, Gershengorn HB, Hua M, et al: Cumulative probability and time to reintubation in U.S. ICUs. Crit Care Med 2017; 45:835-842
2. Thille AW, Richard JC, Brochard L: The decision to extubate in the intensive care unit. Am J Respir Crit Care Med 2013; 187:1294-1302

3. Esteban A, Frutos-Vivar F, Ferguson ND, et al: Noninvasive positivepressure ventilation for respiratory failure after extubation. $N$ Engl J Med 2004; 350:2452-2460

4. Cortegiani A, Accurso G, Mercadante S, et al: High flow nasal therapy in perioperative medicine: From operating room to general ward. BMC Anesthesio/ 2018; 18:166

5. Rochwerg B, Granton D, Wang DX, et al: High flow nasal cannula compared with conventional oxygen therapy for acute hypoxemic respiratory failure: A systematic review and meta-analysis. Intensive Care Med 2019; 45:563-572

6. Groves N, Tobin A: High flow nasal oxygen generates positive airway pressure in adult volunteers. Aust Crit Care 2007; 20:126-131

7. Cortegiani A, Crimi C, Noto A, et al: Effect of high-flow nasal therapy on dyspnea, comfort, and respiratory rate. Crit Care 2019; 23:201

8. Frat JP, Thille AW, Mercat A, et al; FLORALI Study Group; REVA Network: High-flow oxygen through nasal cannula in acute hypoxemic respiratory failure. N Engl J Med 2015; 372:2185-2196

9. Zhu Y, Yin H, Zhang R, et al: High-flow nasal cannula oxygen therapy versus conventional oxygen therapy in patients after planned extubation: A systematic review and meta-analysis. Crit Care 2019; 23:180

10. Ni YN, Luo J, Yu H, et al: Can high-flow nasal cannula reduce the rate of reintubation in adult patients after extubation? A meta-analysis. BMC Pulm Med 2017; 17:142

11. Xu Z, Li Y, Zhou J, et al: High-flow nasal cannula in adults with acute respiratory failure and after extubation: A systematic review and metaanalysis. Respir Res 2018; 19:202

12. Huang HW, Sun XM, Shi ZH, et al: Effect of high-flow nasal cannula oxygen therapy versus conventional oxygen therapy and noninvasive ventilation on reintubation rate in adult patients after extubation: A systematic review and meta-analysis of randomized controlled trials. $J$ Intensive Care Med 2018; 33:609-623

13. Higgins J, Green S: Cochrane Handbook for Systematic Reviews of Interventions Version 5.1.0. The Cochrane Collaboration. 2011. Available at: www.handbook.cochrane.org. Accessed September 9, 2019

14. Guyatt G, Busse J: Risk of Bias in Systematic Reviews. 2011. Available at: http://www.evidencepartners.com/resources/methodologicalresources/risk-of-bias-commentary/. Accessed September 5, 2019

15. Telias I, Ferguson ND: Added benefit of noninvasive ventilation to high-flow nasal oxygen to prevent reintubation in higher-risk patients. JAMA 2019; 322:1455-1457

16. Guyatt $\mathrm{GH}$, Oxman AD, Vist GE, et al; GRADE Working Group: GRADE: An emerging consensus on rating quality of evidence and strength of recommendations. BMJ 2008; 336:924-926

17. Higgins JP, Thompson SG: Quantifying heterogeneity in a meta-analysis. Stat Med 2002; 21:1539-1558

18. Theerawit $P$, Natpobsuk $N$, Sutherasan $Y$ : The efficacy of the Whispherflow CPAP system versus high flow nasal cannula in patients at high risk for postextubation failure. Intensive Care Med Exp 2017:206

19. Rittayamai N, Tscheikuna J, Rujiwit P: High-flow nasal cannula versus conventional oxygen therapy after endotracheal extubation: A randomized crossover physiologic study. Respir Care 2014; 59:485-490

20. Jing G, Li J, Hao D, et al: Comparison of high flow nasal cannula with noninvasive ventilation in chronic obstructive pulmonary disease patients with hypercapnia in preventing postextubation respiratory failure: A pilot randomized controlled trial. Res Nurs Health 2019; 42:217-225

21. Song $H Z, G u J X, X i u H Q$, et al: The value of high-flow nasal cannula oxygen therapy after extubation in patients with acute respiratory failure. Clinics (Sao Paulo) 2017; 72:562-567

22. Maggiore SM, Idone FA, Vaschetto $R$, et al: Nasal high-flow versus Venturi mask oxygen therapy after extubation. Effects on oxygenation, comfort, and clinical outcome. Am J Respir Crit Care Med 2014; 190:282-288

23. Hernández G, Vaquero $C$, González $P$, et al: Effect of postextubation high-flow nasal cannula vs conventional oxygen therapy on 
reintubation in low-risk patients: A randomized clinical trial. JAMA 2016; 315:1354-1361

24. Hernández G, Vaquero C, Colinas L, et al: Effect of postextubation high-flow nasal cannula vs noninvasive ventilation on reintubation and postextubation respiratory failure in high-risk patients: $A$ randomized clinical trial. JAMA 2016; 316:1565-1574

25. Fernandez R, Subira C, Frutos-Vivar F, et al: High-flow nasal cannula to prevent postextubation respiratory failure in high-risk non-hypercapnic patients: A randomized multicenter trial. Ann Intensive Care 2017; 7:47
26. Mauri T, Turrini C, Eronia N, et al: Physiologic effects of high-flow nasal cannula in acute hypoxemic respiratory failure. Am J Respir Crit Care Med 2017; 195:1207-1215

27. Fleeman N, Mahon J, Bates V, et al: The clinical effectiveness and cost-effectiveness of heated humidified high-flow nasal cannula compared with usual care for preterm infants: Systematic review and economic evaluation. Health Technol Assess 2016; 20:1-68

28. Canadian Institute for Health Information: Care in Canadian ICUs. Ottawa, ON, Canada, Canadian Institute for Health Information, 2016 